\title{
ON VECTOR VALUED MULTIPLIERS FOR THE CLASS OF STRONGLY $\mathcal{H}$ K-INTEGRABLE FUNCTIONS
}

\author{
Surinder PAl Singh - SAVita Bhatnagar
}

\begin{abstract}
We investigate the space of vector valued multipliers of strongly Henstock-Kurzweil integrable functions. We prove that if $X$ is a commutative Banach algebra with identity $e$ such that $\|e\|=1$ and $g:[a, b] \longrightarrow X$ is of strongly bounded variation, then the multiplication operator defined by $M_{g}(f):=f g$ maps $\mathcal{S H K}$ to $\mathcal{H} \mathcal{K}$. We also prove a partial converse, when $X$ is a Gel'fand space.
\end{abstract}

\section{Introduction}

A multiplier for a family $\mathcal{A}$ of functions on a compact real interval $[a, b]$ is a function $g$ on $[a, b]$ such that $f g \in \mathcal{A}$, for each $f \in \mathcal{A}$. By Riesz Representation theorem, $L^{\infty}[a, b]$ is precisely the class of multipliers for $L^{1}[a, b]$, see [6].

It is known that real valued continuous functions are not multipliers for the class of real valued Henstock-Kurzweil integrable functions on $[a, b]$. In fact, this set of multipliers is precisely the set of functions of bounded variation on $[a, b]$, see [3, Theorems 6.1.5 and 6.1.9].

This paper aims to study the vector valued multipliers for the family of vector valued strongly Henstock-Kurzweil integrable functions. The case of scalar valued multipliers for the Henstock-Kurzweil integrable functions is already known. Such multipliers for the real valued and for the vector valued Henstock-Kurzweil integrable functions are given in [3] and [5], respectively.

The study of multipliers and the Riesz Representation theorem, for the real valued functions, is related by the fact that if $g$ is a function of bounded variation, then $M_{g}(f):=f g$ defines a multiplication operator from $\mathcal{H} \mathcal{K}$ to $\mathcal{H} \mathcal{K}$ and

(C) 2017 Mathematical Institute, Slovak Academy of Sciences.

2010 Mathematics Subject Classification: Primary 26A39; Secondary 46G10, 28 B05. Keyw ord s: strong Henstock-Kurzweil integrability, bounded variation, Banach algebra, multiplier, $A C G *$ functions, Radon-Nikodym property.

Supported by "DST Purse Grant". 
$T_{g}(f):=(\mathcal{H} \mathcal{K}) \int_{a}^{b} f g$ defines a bounded linear functional on $\mathcal{H} \mathcal{K}$, see [2, Theorems 12.1 and 12.3 ].

Let $X$ be a commutative Banach algebra with identity $e$ such that $\|e\|=1$. We prove that if $g:[a, b] \longrightarrow X$ is of strongly bounded variation, then the multiplication operator defined by $M_{g}(f):=f g$ maps $\mathcal{S H K}$ to $\mathcal{H} \mathcal{K}$, so that $T_{g}$ defined by $T_{g}(f):=(\mathcal{H} \mathcal{K}) \int_{a}^{b} f g$ is a bounded linear operator from $\mathcal{S H K}$ to $X$.

We prove the converse under some restrictions. For that we require $X$ to be a Gel'fand space, which is equivalent to the requirement that $X$ satisfies the Radon-Nikodym property with respect to the Lebesgue measure on $[a, b]$. In that case, we prove that if $T: \mathcal{S H K} \longrightarrow X$ is a bounded linear operator, then there exists some $g \in{ }^{*} \mathcal{B} \mathcal{V}$ such that $\tau(T(f))=(\mathcal{H K}) \int_{a}^{b} \tau(f g)$ holds for all $f \in \mathcal{S H K}$ and for all multiplicative linear functionals $\tau$ on $X$.

If $X=\mathbb{R}$, then our results are reduced to the Riesz Representation theorem and the related multipliers for the class of Henstock-Kurzweil integrable functions.

\section{Preliminaries}

Let $[a, b]$ be a compact real interval, $\mathcal{I}$ be the family of compact subintervals of $[a, b]$ and $X$ be a commutative Banach algebra with identity $e$ of norm 1 .

A function $F: \mathcal{I} \longrightarrow X$ is said to be additive if $F(J \cup K)=F(J)+F(K)$, for all non-overlapping intervals $J, K \in \mathcal{I}$ such that $J \cup K \in \mathcal{I}$.

A collection $\left\{\left(t_{i}, J_{i}\right) ; i=1, \ldots, k\right\}$ of point-interval pairs is called a tagged-partition of the interval $[a, b]$ if each $t_{i} \in J_{i}$ and $\left\{J_{i}: i=1, \ldots, k\right\}$ are pairwise non-overlapping compact subintervals of $[a, b]$ with $[a, b]=\bigcup_{i=1}^{k} J_{i}$.

Any positive function $\delta:[a, b] \longrightarrow(0,+\infty)$ is called a gauge on $[a, b]$ and the above tagged partition is said to be $\delta$-fine if $J_{i} \subset\left(t_{i}-\delta\left(t_{i}\right), t_{i}+\delta\left(t_{i}\right)\right)$, for every $i=1, \ldots, k$.

A function $f:[a, b] \longrightarrow X$ is said to be strongly Henstock-Kurzweil integrable on $[a, b]$, if there is an additive function $F: \mathcal{I} \longrightarrow X$ such that for every $\epsilon>0$ there exists a gauge $\delta$ on $[a, b]$ such that the inequality

$$
\sum_{i=1}^{k}\left\|f\left(t_{i}\right) \mu\left(J_{i}\right)-F\left(J_{i}\right)\right\|_{X}<\epsilon
$$

is satisfied for every $\delta$-fine tagged partition $\left\{\left(t_{i}, J_{i}\right): i=1, \ldots, k\right\}$ of $[a, b]$.

The $\mathcal{H} \mathcal{K}$-integral is defined on the same lines, except the summation sign in (2.1) comes inside the norm sign. In that case, $F([a, b])$ is known as the Henstock-Kurzweil integral of $f$ over an interval $[a, b]$ and is denoted by $(\mathcal{H K}) \int_{a}^{b} f \mathrm{~d} \mu$. For more details on these integrals, see [7]. 


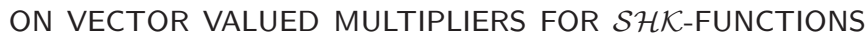

The classes of the strongly Henstock-Kurzweil and the Henstock-Kurzweil integrable functions from $[a, b]$ to $X$ are denoted by $\mathcal{S H K}$ and $\mathcal{H} \mathcal{K}$, respectively. If $X$ is a finite dimensional space, then $\mathcal{S H K}=\mathcal{H} \mathcal{K}$. In general, $\mathcal{S H K} \subseteq \mathcal{H} \mathcal{K}$, for more details, see 7 .

It is easy to see that the product of an $\mathcal{S H K}$ function and a vector valued continuous function may not be in $\mathcal{S H K}$. For example, take

$$
f(t):=\sum_{n=1}^{\infty} 2^{n} c_{n} \chi_{I_{n}}(t) e,
$$

where $\Sigma c_{n}$ is a non-absolutely convergent series for which $\sum\left|c_{n}\right| / \sqrt{n}$ does not converge and each $I_{n}=\left(2^{-n}, 2^{-n+1}\right)$. Take $g$ to have the value $2\left(\operatorname{sgn} c_{n}\right) e / \sqrt{n}$ at the midpoint of each $I_{n}$, the value zero at the endpoints of each $I_{n}$ and at 0 , and linear on the rest of $[0,1]$. Then, it can be shown that $f g \notin \mathcal{S H} \mathcal{K}$.

Definitions 2.1. Let $F:[a, b] \longrightarrow X$ be a given function.

(1) $F$ is said to be of strongly bounded variation $(\mathcal{B V})$ on $[a, b]$ if

$$
\sup \sum_{i}\left\|F\left(d_{i}\right)-F\left(c_{i}\right)\right\|_{X}<\infty
$$

where the supremum is taken over all finite collections of non-overlapping intervals $\left\{\left[c_{i}, d_{i}\right]\right\}$ in $[a, b]$.

(2) $F$ is said to be of bounded variation $\left({ }^{*} \mathcal{B V}\right)$ on $[a, b]$ if

$$
\sup \left\|\sum_{i}\left[F\left(d_{i}\right)-F\left(c_{i}\right)\right]\right\|_{X}<\infty,
$$

where the supremum is taken over all finite collections of non-overlapping intervals $\left\{\left[c_{i}, d_{i}\right]\right\}$ in $[a, b]$.

(3) $F$ is said to be strongly absolutely continuous $(\mathcal{A C})$ on $[a, b]$ if for each $\epsilon>0$ there exists $\eta>0$ such that

$$
\sum_{i}\left\|F\left(d_{i}\right)-F\left(c_{i}\right)\right\|_{X}<\epsilon
$$

for all finite collections of non-overlapping intervals $\left\{\left[c_{i}, d_{i}\right]\right\}$ in $[a, b]$ satisfying $\Sigma_{i}\left(d_{i}-c_{i}\right)<\eta$.

Definition 2.2. A function $F:[a, b] \longrightarrow X$ is said to be of strong bounded slope variation on $[a, b]$ if

$$
\sup \sum_{i=1}^{n}\left\|\frac{F\left(x_{i+1}\right)-F\left(x_{i}\right)}{x_{i+1}-x_{i}}-\frac{F\left(x_{i}\right)-F\left(x_{i-1}\right)}{x_{i}-x_{i-1}}\right\|_{X}<\infty,
$$

where the supremum is taken over all partitions $a=x_{0}<x_{1}<\cdots<x_{n+1}=b$ of $[a, b]$. As usual, a function $F:[a, b] \longrightarrow X$ is said to be of weak bounded 
slope variation on $[a, b]$ if for every $x^{*} \in X^{*}$, the real valued function $x^{*} \circ F$ is of bounded slope variation on $[a, b]$.

Definitions 2.3. Let $F:[a, b] \longrightarrow X$ be a given function.

(1) The oscillation of $F$ on $[c, d] \subset[a, b]$ is defined as

$$
w(F,[c, d]):=\sup _{[\alpha, \beta] \subseteq[c, d]}\|F(\beta)-F(\alpha)\|_{X} .
$$

(2) $F$ is said to be $\mathcal{A C}_{*}$ on a set $E \subset[a, b]$ if

$$
\sum_{i} w\left(F,\left[c_{i}, d_{i}\right]\right)<\epsilon
$$

for all finite collections $\left\{\left[c_{i}, d_{i}\right]\right\}$ of non-overlapping intervals that have endpoints in $E$ and satisfy $\Sigma_{i}\left(d_{i}-c_{i}\right)<\eta$.

(3) $F$ is said to be $\mathcal{A C G}_{*}$ on $[a, b]$ if $[a, b]$ can be decomposed as a countable union of sets on each of which $F$ is $\mathcal{A C}_{*}$.

The classes of $\mathcal{B V},{ }^{*} \mathcal{B V}, \mathcal{A C}, \mathcal{A C}_{*}$ and $\mathcal{A C G}_{*}$ functions over $[a, b]$ are denoted themselves by $\mathcal{B V},{ }^{*} \mathcal{B V}, \mathcal{A C}, \mathcal{A C}_{*}$ and $\mathcal{A C G}_{*}$, respectively. In case of real valued functions, the class $\mathcal{B} \mathcal{V}$ will be denoted by $\mathcal{B} \mathcal{V}_{\mathbb{R}}$.

Clearly, $\mathcal{A C}_{*} \subset \mathcal{A C} \subset \mathcal{B V}$. Moreover, the collections $\mathcal{A C}$ and $\mathcal{A C}_{*}$ are the same, when $E$ is an interval. For more details on these integrals, see [7].

As usual, a function $F:[a, b] \longrightarrow X$ is said to be of weakly bounded variation if $x^{*} \circ(F) \in \mathcal{B} \mathcal{V}$, for each function $x^{*} \in X^{*}$.

In [7, Theorem 7.1.9], the following result is proved.

Theorem 2.4. Let $F:[a, b] \longrightarrow X$ be a given function. Then, $F$ is ${ }^{*} \mathcal{B V}$ if and only if $F$ is of weakly bounded variation on $[a, b]$.

For more on the above notions $\mathcal{A C G}_{*}, \mathcal{S H K}, \mathcal{B V}$ for vector valued functions, the reader may refer to [7].

As $X$ is a commutative Banach algebra with identity $e$ of norm 1 , by $[6$, Theorem 18.3], every proper ideal of $X$ is contained in a maximal ideal, and every maximal ideal is closed.

Let $\Delta$ denote the set of all non-zero multiplicative linear functionals (MLFs) of $X$. Since $X$ has an identity, we have $\Delta \neq \emptyset$. Indeed, by [ $[$, Theorem 18.17], there is a one-to-one correspondence between $\Delta$ and the class of maximal ideals in the sense that every maximal ideal is the kernel of some $\tau \in \Delta$, and conversely, the kernel of every $\tau(\in \Delta)$ is the maximal ideal associated with $\tau$.

We now present a result on integration by parts for $\mathcal{S H \mathcal { K }}$ functions. It is a generalization of [3, Lemma 6.1.2]. 
Lemma 2.1. Let $f \in \mathcal{S H \mathcal { K }}$ and $\phi \in L^{1}([a, b], X)$. Then, the map $s \longrightarrow f(s) \int_{a}^{s} \phi$ belongs to $\mathcal{H K}$ and

$$
(\mathcal{H} \mathcal{K}) \int_{a}^{b}\left\{f(s) \int_{a}^{s} \phi(t) \mathrm{d} t\right\} \mathrm{d} s=\int_{a}^{b}\left\{(\mathcal{H} \mathcal{K}) \int_{s}^{b} f(t) \mathrm{d} t\right\} \phi(s) \mathrm{d}(s) .
$$

Proof. Let $F(s)=(\mathcal{H} \mathcal{K}) \int_{a}^{s} f(t) \mathrm{d} t ; s \in[a, b]$ and let $\epsilon>0$ be given. By Saks-Henstock lemma, there is a gauge $\delta_{1}$ on $[a, b]$ such that

$$
\sum_{(t,[u, v]) \in P)}\|f(t)(v-u)-[F(v)-F(u)]\|_{X}<\epsilon,
$$

holds, for each $\delta_{1}$-fine partial division $P$ of $[a, b]$.

Also, by [7, Theorem 7.4.1], $F$ is continuous on $[a, b]$ and thus, uniformly continuous on $[a, b]$. Choose $\delta_{2}>0$ such that $\|F(\beta)-F(\alpha)\|<\epsilon$ holds for every subinterval $[\alpha, \beta] \subset[a, b]$ with $0<\beta-\alpha<\delta_{2}$.

Define a gauge $\delta$ on $[a, b]$ by setting $\delta(x):=\min \left\{\delta_{1}(x), \delta_{2}\right\}$, for all $x \in[a, b]$. Let $P$ be any $\delta$-fine tagged-partition of the interval $[a, b]$.

Let $g(s):=\int_{a}^{s} \phi$, for all $s \in[a, b]$ and let $M:=\int_{a}^{b}\|\phi\|_{X}$ be the $L_{1}$-norm of $\phi$. Note that $F(b) g(b)=\sum_{(t,[u, v]) \in P}[F(v) g(v)-F(u) g(u)]$ and we have

$$
\begin{aligned}
& \left\|\sum_{(t,[u, v]) \in P} f(t) g(t)(v-u)-\int_{a}^{b}\left(\mathcal{H} \mathcal{K} \int_{s}^{b} f(t) \mathrm{d} t\right) \phi(s) \mathrm{d} s\right\|_{X} \\
= & \sum_{(t,[u, v]) \in P}[f(t) g(t)(v-u)-[F(v) g(v)-F(u) g(u)]] \\
& +\int_{a}^{b}\left(F(b)-(\mathcal{H} \mathcal{K}) \int_{s}^{b} f(t) \mathrm{d} t\right) \phi(s) \mathrm{d} s \|_{X} \\
= & \sum_{(t,[u, v]) \in P}\left[f(t) g(t)(v-u)-[F(v) g(v)-F(u) g(u)]+\int_{u}^{v} F(s) \phi(s) \mathrm{d} s\right] \|_{X} \\
\leq & \left\|\sum_{(t,[u, v]) \in P}[f(t) g(t)(v-u)-[F(v)-F(u)] g(t)]\right\|_{X} \\
& +\left\|\sum_{(t,[u, v]) \in P}\left[[F(v)-F(u)] g(t)-[F(v) g(v)-F(u) g(u)]+\int_{u}^{v} F \phi\right]\right\|_{X} \\
\leq & \left\|\sum_{(t,[u, v]) \in P}\left(f(t)(v-u)-(\mathcal{H} \mathcal{K}) \int_{u}^{v} f\right) \int_{a}^{t} \phi\right\|_{X}
\end{aligned}
$$




$$
\begin{aligned}
& +\left\|\sum_{(t,[u, v]) \in P}\left[[F(v)-F(u)] \int_{v}^{t} \phi+\int_{u}^{v}[F-F(u)] \phi\right]\right\|_{X} \\
& \leq M \epsilon+\epsilon \sum_{(t,[u, v]) \in P}\left[\int_{v}^{t}\|\phi\|_{X}+\int_{u}^{v}\|\phi\|_{X}\right] \leq 3 M \epsilon .
\end{aligned}
$$

Since $\epsilon>0$ is arbitrary, the result follows.

Lemma 2.2. Let $g:[a, b] \longrightarrow X$ be such that $g \in \mathcal{B V}$. Then, there is a sequence $\left\{\phi_{n}\right\}$ of $X$-valued step functions on $[a, b]$ such that $\sup _{n}\left\|\phi_{n}\right\|_{1, X}<\infty$ and

$$
\lim _{n \rightarrow \infty} \int_{a}^{t} \phi_{n} \mathrm{~d} \mu=g(t)-g(a) \quad \text { for all } \quad t \in[a, b] .
$$

Proof. The proof is analogous to the real case, as in [3, Theorem 6.1.4].

LEMma 2.3. Let $f \in \mathcal{S H K}$ and $\left\{\phi_{n}\right\}$ be a sequence in $L^{1}([a, b], X)$ such that $\sup _{n \in \mathbb{N}}\left\|\phi_{n}\right\|_{1, X}<\infty$. Let $g_{n}(s):=\int_{a}^{s} \phi_{n}(t) d t$ for all $s \in[a, b]$. Then, the sequence $\left\{f g_{n}\right\}$ is $\mathcal{H} \mathcal{K}$-equi-integrable on $[a, b]$.

Proof. Imitate the proof of Lemma 2.1 and obtain a gauge independent of $n$.

LEMma 2.4. Under the hypothesis of Lemma 2.3, if the sequence $\left\{g_{n}\right\}$ is pointwise convergent on $[a, b]$, then $\lim _{n \longrightarrow \infty} f g_{n} \in \mathcal{H} \mathcal{K}$ and

$$
(\mathcal{H K}) \int_{a}^{b} \lim _{n \longrightarrow \infty} f g_{n}=\lim _{n \longrightarrow \infty}(\mathcal{H K}) \int_{a}^{b} f g_{n} .
$$

Proof. See [7, the page 65, Theorem 3.5.2].

Remark 1. For each $f \in \mathcal{S H} \mathcal{K}$, if we define

$$
\|f\|_{\mathcal{H} \mathcal{K}}:=\sup \left\{\left\|(\mathcal{H K}) \int_{I} f\right\|_{X}: I \in \mathcal{I}\right\}
$$

then $\|\cdot\|_{\mathcal{H} \mathcal{K}}$ is a semi-norm on $\mathcal{S H} \mathcal{K}$.

Further, if we define a relation $\sim$ on $\mathcal{S H K}$ as $f \sim g$ if $f=g$ a.e., then $\sim$ is an equivalence relation on $\mathcal{S H \mathcal { K }}$. Therefore, $\mathcal{S H} \mathcal{K} / \sim$ is a normed linear space, which is not a Banach space even for $X=\mathbb{R}$. 
Theorem 2.5. If $f \in \mathcal{S H K}$ and $g \in \mathcal{B V}$, then $f g \in \mathcal{H} \mathcal{K}$. Moreover, in this case,

$$
\left\|(\mathcal{H} \mathcal{K}) \int_{a}^{b} f g\right\| \leq\|f\|_{\mathcal{H} \mathcal{K}}\{\|g(a)\|+\operatorname{Var}(g,[a, b])\} .
$$

Pr o of. The proof follows from Lemma 2.1, Lemma 2.2 and Lemma 2.3, reasoning as in Theorem 6.1.8 in [3, page 173].

Hence, any $g \in \mathcal{B} \mathcal{V}$ corresponds to the multiplication operator $M_{g}: \mathcal{S H} \mathcal{K} \longrightarrow \mathcal{H} \mathcal{K}$ given by $M_{g}(f):=f g$ for all $f \in \mathcal{S H} \mathcal{K}$.

Also, the corresponding integral operator

$$
T_{g}: \mathcal{S H K} \longrightarrow X \text { given by } T_{g}(f):=(\mathcal{H} \mathcal{K}) \int_{a}^{b} f g
$$

is a bounded linear operator, which is analogous to the Riesz representation theorem for the vector valued case.

\section{The problem with multipliers}

The question, whether every multiplier of $\mathcal{S H} \mathcal{K}$ is given by a function $g \in \mathcal{B V}$, remains unresolved. The following are associated problems:

(1) Let $T: \mathcal{S H K} \longrightarrow X$ be a bounded linear operator. Can we find some $g \in \mathcal{B V}$ such that $T(f)=(\mathcal{H} \mathcal{K}) \int f g$, for all $f \in \mathcal{S H} \mathcal{K}$ ?

(2) Let $M: \mathcal{S H K} \longrightarrow \mathcal{H} \mathcal{K}$ be a multiplication operator. Can we find some $g \in \mathcal{B} \mathcal{V}$ such that $M(f)=f g$, for all $f \in \mathcal{S H} \mathcal{K}$ ?

From [3], following the scalar case, define

$$
C_{\mathcal{S H K}}:=\left\{F: \text { there is } f \in \mathcal{S H K} \text { such that } F(t)=\int_{t}^{b} f \text {, for all } t \in[a, b]\right\} .
$$

Then, $C_{\mathcal{S H K}}$ is a linear subspace of $C([a, b], X)$. Define $T_{0}: C_{\mathcal{S H K}} \rightarrow X$ by $T_{0}(F):=T(f)$. Then $T_{0}$ is a bounded linear operator on the linear subspace $C_{\mathcal{S H K}}$ of $C([a, b], X)$. The question is:

Can $T_{0}$ be lifted to $C([a, b], X)$ while preserving norm? 
That is, whether Hahn-Banach type theorem is true for such bounded linear operators. The answer is NO. In fact, the operators between Banach spaces, for which such norm preserving extensions are possible, are called Hahn-Banach operators, see 4. In general, such a result is not true.

In [2], this converse problem for $X=\mathbb{R}$, that is, the multipliers of $\mathcal{H} \mathcal{K}$ integrable functions are of bounded variation, has been proved without using Hahn-Banach extensions. By these proofs, we encounter the following problem in Banach-valued functions:

A strongly absolutely continuous function may not be differentiable.

For more details, see [7, Example 7.3.9]. To remedy the above problem, we put certain restrictions on the Banach algebra $X$. We have the following notions and results from [1, pages 61,107$]$.

\section{DeFinitions 3.1.}

(1) A Banach space $X$ is said to have the Radon-Nikodym property $(\mathcal{R} \mathcal{N} \mathcal{P})$ with respect to the finite measure space $(\Omega, \Sigma, \mu)$ if for each $\mu$-continuous vector measure $G: \Sigma \rightarrow X$ of strongly bounded variation, there exists $g \in L^{1}(\mu, X)$ such that

$$
G(E)=\int_{E} g \mathrm{~d} \mu, \quad \text { for all } \quad E \in \Sigma \text {. }
$$

(2) A Banach space $X$ is said to be a Gel'fand space if each absolutely continuous function $f:[a, b] \rightarrow X$ is differentiable almost everywhere.

ThEOREM 3.2. A Banach space $X$ is a Gel'fand space if and only if $X$ has the $\mathcal{R N} \mathcal{P}$ with respect to the Lebesgue measure on the Borel sets in $[a, b]$.

Proof. See [1, Theorem 2, page 107].

Remark 2. It can be seen from the proof of the above theorem that if $X$ has $\mathcal{R N} \mathcal{P}$ then the derivative of an absolutely continuous function is a Bochner integrable function.

Spaces that have $\mathcal{R} \mathcal{N} \mathcal{P}$ include reflexive spaces, separable duals, spaces with a bounded complete basis, $L^{p}(\mu, X)$, if $X$ has $\mathcal{R N} \mathcal{P}$ and $1<p<\infty$. However, spaces $L^{1}[0,1], B V_{0}[0,1], c_{0}, c, \ell^{\infty}, L^{\infty}[0,1], L^{1}(\mu), \mu$ not purely atomic, $C(\Omega), \Omega$ infinite compact $T_{2}$-space, $\mathcal{K}(X)$-compact operators on $X=\ell^{p}, L^{p}, C(\Omega)$ do not have $\mathcal{R} \mathcal{N} \mathcal{P}$, see [1, p. 218]. 


\section{ON VECTOR VALUED MULTIPLIERS FOR $\mathcal{S H K}$-FUNCTIONS}

\section{The main result}

Theorem 4.1. If the Banach algebra $X$ is a Gel'fand space and $T: \mathcal{S H K} \rightarrow X$ is a bounded linear operator, then there exists $g \in{ }^{*} \mathcal{B V}$ such that

$$
\tau(T(f))=(\mathcal{H} \mathcal{K}) \int_{a}^{b} \tau(f) \tau(g), \quad \text { for all } \quad f \in \mathcal{S H K} \text { and } \tau \in \Delta .
$$

P r o o f. Define $G(t):=T\left(\chi_{[a, t]} e\right)$, for all $t \in[a, b]$. We complete the proof in four steps, as below.

Step I: The function $G$ satisfies the Lipschitz condition and hence is absolutely continuous on $[a, b]$. This is true, as for any $[c, d] \subset[a, b]$, we have

$$
\|G(d)-G(c)\|=\left\|T\left(\chi_{(c, d]} e\right)\right\| \leq\|T\|\left\|\chi_{(c, d]} e\right\|_{\mathcal{H} \mathcal{K}}=\|T\|(d-c) .
$$

Since $X$ is Gel'fand space and $G \in \mathcal{A C}$, it is differentiable almost everywhere. Let $g(t):=G^{\prime}(t)$, almost everywhere on $[a, b]$.

Step II: The function $G$ is of weak bounded slope variation.

Let $x^{*} \in X^{*}$. For any partition $\left\{y_{0}, y_{1}, \ldots, y_{m+1}\right\}$ of $[a, b]$, we have

$$
\begin{aligned}
\sum_{i=1}^{m}\left|x^{*}\left(\frac{G\left(y_{i+1}\right)-G\left(y_{i}\right)}{y_{i+1}-y_{i}}-\frac{G\left(y_{i}\right)-G\left(y_{i-1}\right)}{y_{i}-y_{i-1}}\right)\right| & =\sum_{i=1}^{m}\left|x^{*}\left(T\left(\phi_{i}\right)\right)\right| \\
& =\sum_{i=1}^{m} x^{*}\left(T\left(e_{i} \phi_{i}\right)\right)
\end{aligned}
$$

where $e_{i}$ is either $e$ or $-e$, as the case may be, and

$$
\phi_{i}:=\frac{\chi_{\left(y_{i}-y_{i+1}\right]}}{y_{i+1}-y_{i}}-\frac{\chi_{\left(y_{i-1}-y_{i}\right]}}{y_{i}-y_{i-1}} .
$$

Since $T$ is bounded, so is $x^{*} \circ T$. Therefore, (4.2) becomes

$$
\left(x^{*} \circ T\right)\left(\sum_{i=1}^{m} e_{i} \phi_{i}\right) \leq\left\|x^{*} \circ T\right\|\left\|\sum_{i=1}^{m} e_{i} \phi_{i}\right\|_{\mathcal{H \mathcal { K }}} \leq 2\left\|x^{*} \circ T\right\| \leq 2\left\|x^{*}\right\|\|T\| .
$$

This proves that $x^{*} \circ G$ is of bounded slope variation, for all $x^{*} \in X^{*}$. Hence, $G$ is of weak bounded slope variation.

Step III: For all step functions $\phi:[a, b] \rightarrow X$ and for all $x^{*} \in X^{*}$, we have

$$
x^{*}(T(\phi))=(\mathcal{H} \mathcal{K}) \int_{a}^{b} x^{*}(\phi g) .
$$

Fix any $x^{*} \in X^{*}$. Since the function $G$ satisfies the Lipschitz condition, so does the function $x^{*} \circ G$. Also $x^{*} \circ G$ is of bounded slope variation. 


\section{SURINDER PAL SINGH — SAVITA BHATNAGAR}

By Lemma 12.6 from [2], $x^{*} \circ G$ is the primitive of some scalar function from $\mathcal{B} \mathcal{V}_{\mathbb{R}}$, say $g_{x^{*}} \in \mathcal{B} \mathcal{V}_{\mathbb{R}}$.

Therefore, $\left(x^{*} \circ G\right)(t)=\int_{a}^{t} g_{x^{*}}(s) \mathrm{d} s$, for all $t \in[a, b]$, which implies $\left(x^{*} \circ G\right)^{\prime}=g_{x^{*}}$, a.e. on $[a, b]$. Note that $\left(x^{*} \circ G\right)^{\prime}=x^{*} \circ g$, whenever $G^{\prime}=g$. Thus $g_{x^{*}}=x^{*}(g)$, a.e. on $[a, b]$.

The linearity of $x^{*}$ ensures (4.3), for all step functions $\phi:[a, b] \rightarrow X$.

Step IV: The result 4.1 holds.

Let $f \in \mathcal{S H} \mathcal{K}$. We first show that there is a sequence $\left\{\varphi_{n}\right\}$ of $\mathrm{X}$-valued step functions weakly control convergent to $f$.

Applying [7, Theorem 7.4.3], the primitive $F$ of $f$ is $\mathcal{A C G}_{*}$ on $[a, b]$. That is, $[a, b]$ is a countable union of closed sets $\left\{X_{i}\right\}$, on each of which $F$ is $\mathcal{A C}_{*}$. For convenience, assume that $a, b \in X_{i}$ for all $i$. For each $n \in \mathbb{N}$, let $F_{n}(x)=F(x)$ when $x \in X_{1} \cup \ldots \cup X_{n}$ and linear elsewhere. Then, $F_{n}$ is $\mathcal{A C}_{*}$ on $[a, b]$ and hence, $\mathcal{A C}$ on $[a, b]$, see [7, Theorem 7.1.11].

Since $X$ is a Gel'fand space, each $F_{n}$ is differentiable almost everywhere. For each $n \in \mathbb{N}$, put $f_{n}(x):=F_{n}^{\prime}(x)$, almost everywhere on $[a, b]$. Therefore, there is a subsequence $\left\{f_{n_{k}}\right\}$ of $\left\{f_{n}\right\}$, which is weakly control convergent to $f$ on $[a, b]$.

Also by Remark 2, each $f_{n}$ is Bochner integrable on $[a, b]$. Therefore, for each $n$, there are $X$-valued simple functions and hence $X$-valued step functions $\varphi_{n}$ satisfying

$$
\int_{a}^{b}\left\|f_{n}-\varphi_{n}\right\|_{X}<2^{-n}
$$

Thus, $\sum_{n=1}^{\infty}\left\|f_{n}(t)-\varphi_{n}(t)\right\|$ converges almost everywhere and is strongly Henstock-Kurzweil integrable on $[a, b]$. As $\varphi_{n}=\left[\varphi_{n}-f_{n}\right]+f_{n}$, we have $\left\{\varphi_{n}\right\} \longrightarrow f$, a.e. on $[a, b]$. Since

$$
\left\|\int_{u}^{v} \varphi_{n}\right\| \leq \int_{u}^{v}\left\|f_{n}-\varphi_{n}\right\|+\left\|\int_{u}^{v} f_{n}\right\|
$$

the subsequence $\left\{\varphi_{n_{k}}\right\}$ of $\left\{\varphi_{n}\right\}$ is weakly control convergent to $f$ on $[a, b]$. Now, if $\tau \in \Delta$, then, by [2, Theorem 12.4], we obtain

$$
\tau(T(f))=\lim _{n \rightarrow \infty} \tau\left(T\left(\varphi_{n}\right)\right)=\lim _{n \rightarrow \infty}(\mathcal{H} \mathcal{K}) \int_{a}^{b} \tau\left(\varphi_{n}\right) \tau(g)=(\mathcal{H} \mathcal{K}) \int_{a}^{b} \tau(f) \tau(g) .
$$

Hence, the result.

This also proves the following result. 


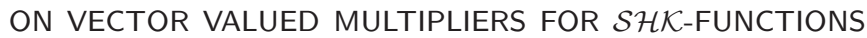

TheOREM 4.2. If the Banach algebra $X$ is a Gel'fand space and $M: \mathcal{S H K} \longrightarrow$ $\mathcal{H} \mathcal{K}$ is a bounded linear multiplication operator, then there exists $g \in{ }^{*} \mathcal{B} \mathcal{V}$ such that

$$
\tau(M(f))=\tau(f g), \quad \text { for all } f \in \mathcal{S H} \mathcal{K} \text { and } \tau \in \Delta .
$$

In other words,

\section{Concluding Remarks 4.3.}

$$
\tau(M)=\tau\left(M_{g}\right)
$$

(1) If $f \in \mathcal{H} \mathcal{K}$, then its primitive is only * $\mathcal{A C G}$ and not $\mathcal{A C G}_{*}$, so the above proof works only for $f \in \mathcal{S H} \mathcal{K}$. For ${ }^{*} \mathcal{A C G}$ functions, see [7].

(2) What is the solution to the problem in case of strongly McShane integrable functions when $\operatorname{dim} X$ is infinite or the case of functions of several variables?

Acknowledgements. The authors are grateful to (retired) Professor Ajit Iqbal Singh of University of Delhi for her helpful suggestions and continuous encouragement.

\section{REFERENCES}

[1] DieSteL, J.-UHL, J. J.: Vector Measures, in: Math. Surveys Monogr., Vol. 15, Amer. Math. Soc., Providence, R.I., 1977.

[2] LEE, PENG YEE: Lanzhou Lectures on Henstock Integration, in: Ser. Real Anal., Vol. 2, World Sci. Publ. Co., Singapore, 1989.

[3] LEE, TUO-YEONG: Henstock-Kurzweil Integration on Euclidean Spaces, in: Ser. Real Anal., Vol. 12, World Sci. Publ. Co., Singapore, 2011.

[4] OStRovSKII, M. I.: Hahn Banach operators, Proc. Amer. Math. Soc. 129 (2001), 2923-2930.

[5] DI PIAZZA, L.-MARRAFFA, V.: The Mcshane, PU and Henstock integrals of Banach valued functions, Czechoslovak Math. J. 52 (2002), 609-633.

[6] RUDIN, W.: Real and Complex Analysis (3rd ed.), McGraw Hill, New York, 1987.

[7] SCHWABIK, Š-_GUOJU, YE: Topics in Banach Space Integration, in: Ser. Real Anal., Vol. 10, World Sci. Publ. Co., Singapore, 2005.

Center for Advanced Study in Mathematics

Department of Mathematics

Panjab University Chandigarh

160014 Chandigarh

INDIA

E-mail: sps@pu.ac.in bhsavita@pu.ac.in 